\title{
VIDEO-ASSISTED THORACOSCOPIC SURGERY IN PATIENTS PREVIOUSLY TREATED FOR INTRATHORACIC LYMPHOMA
}

\author{
Tiziano De Giacomo, MD, Erino Angelo Rendina, MD, Federico Venuta, MD, Isacco Flaishman, MD, and \\ Costante Ricci, MD, Rome, Italy
}

The mediastinum, a frequent site of lymphoma, is involved in $50 \%$ to $60 \%$ of cases of Hodgkin's disease and $20 \%$ of cases of non-Hodgkin lymphoma. ${ }^{1}$ In recent years the curability rate has reached $80 \%$ for Hodgkin's disease and $50 \%$ to $70 \%$ for non-Hodgkin lymphoma ${ }^{2}$ as a result of improved therapeutic regimens, careful monitoring of response, and treatment of relapses. Residual pathologic tissue in the mediastinum and the presence of lung lesions are frequent findings after chemotherapy or radiation therapy. Such findings may stem from relapsing disease, fibrosis, or new and different pathology, so surgical biopsy is often necessary to achieve an unequivocal diagnosis.

Some of these lesions can be approached with mediastinoscopy or through an anterior mediastinotomy, ${ }^{3,4}$ but for others only invasive procedures (partial or total sternotomy or thoracotomy) can be undertaken. ${ }^{5}$ Patients treated for lymphoma are often in poor general condition, and less invasive procedures are preferable. Video-assisted thoracoscopic surgery (VATS) seems to fulfill these requirements, and we have employed it as an alternative to sternotomy and thoracotomy for biopsy and resection in these patients. Between April 1992 and November 1995, 32 patients (18 male and 14 female) previously treated for lymphoma showed evidence of mediastinal or pulmonary lesions and underwent VATS. The mean age was 34.5 years (range 15 to 68 years).

Patients whose conditions could be managed with mediastinoscopy or anterior mediastinotomy, according to previously reported criteria, ${ }^{4}$ were excluded from this group. Mediastinoscopic examination was inconclusive in five cases, however, and these patients underwent VATS successfully. Thirteen patients were affected by Hodgkin's disease and 19 were affected by non-Hodgkin lymphoma. Fourteen patients had mediastinal masses, eight had lung lesions, and 10 had combined mediastinal and lung lesions. In five cases, massive pleural effusion was present; in two of the five cases, concomitant pericardial effusion was present. Noninvasive diagnostic procedures, such as serologic tests, bone marrow biopsy, sputum culture or cytologic examination, bronchoalveolar lavage, cytologic examination and culture of pleural

From the Department of Thoracic Surgery, University of Rome "La Sapienza," Rome, Italy.

Received for publication Feb. 15, 1996; accepted for publication Feb. 29, 1996.

J Thorac Cardiovasc Surg 1996;112:1108-9

Copyright (C) 1996 by Mosby-Year Book, Inc.

$0022-5223 / 96 \$ 5.00+0 \quad \mathbf{1 2 / 5 4 / 7 3 2 3 5}$
Table I. Associated preoperative conditions

\begin{tabular}{llc}
\hline & \multicolumn{1}{c}{ Definition } & No. of patients \\
\hline Anemia & $\mathrm{Hb}<8 \mathrm{gm} / \mathrm{dl}$ & 5 \\
Thrombocytopenia & $<5000$ cells $/ \mu \mathrm{g}$ & 3 \\
Granulocytopenia & $<2000$ cells $/ \mu \mathrm{g}$ & 4 \\
Weight loss & $>5 \mathrm{~kg} \mathrm{loss}$ & 7 \\
$\begin{array}{l}\text { Impairment of respi- } \\
\quad \text { ratory function }\end{array}$ & $\mathrm{FEV}_{1}<50 \%$ & 6 \\
\hline $\mathrm{Hb}$, Hemoglobin; $F E V_{I}$, forced expiratory volume in 1 second.
\end{tabular}

and pericardial effusion, and fine-needle biopsy, had inconclusive results. Preoperative evaluation included complete blood cell count, coagulation tests, blood gas analysis, chest radiography, fiberoptic bronchoscopy, and computed tomography of the chest. Preoperative associated conditions are reported in Table I. Three patients with severe thrombocytopenia $(<50000 \mathrm{cells} / \mu \mathrm{l})$ required platelet transfusions before the operation. Thoracoscopy was performed according to a previously described technique. ${ }^{4}$

Mean operative time was 52 minutes (15 to 80 minutes). There were no intraoperative complications. No patients required postoperative blood transfusions. In eight cases we removed the residual mediastinal tissue completely. In one of the eight, a concomitant pleuropericardial cyst was removed as well. A pericardial window was made in two patients with recurrent pericardial effusion from relapsing lymphoma. Pleural localization of lymphoma was demonstrated in five patients with a recurrent, cytopathologically negative effusion. Procedures performed and histologic findings are reported in Table II. Eighteen patients (50\%) had recurrent lymphoma, and the remaining 17 had either new lesions or residual fibrosis. The latter group of patients was followed up (range 3 to 41 months) without evidence of recurrence. In one patient, frozen sections showed evidence of adenocarcinoma, and we converted thoracoscopy to thoracotomy for left upper lobectomy. Three patients had superficial infections of the trocar site. Mean postoperative hospitalization was 5 days (range 2 to 14 days) and the mean duration of chest tube drainage was 3 days (range 1 to 6 days). The presence of a residual mediastinal mass or of new pulmonary or mediastinal lesions is a common finding in patients previously treated for lymphoma, and surgical biopsy or resection is required if noninvasive procedures do not yield unequivocal diagnosis. The surgical approach can present some clinical and technical problems in this group of patients. At the primary site of disease, the marked reduction of the 
Volume 112, Number 4

Table II. Thoracoscopic procedures and histologic findings

\begin{tabular}{lc}
\hline & No. of patients \\
\hline Procedure & \\
Biopsy of mediastinal lesions & 16 \\
Resection of mediastinal lesions & 8 \\
Lung biopsy & 5 \\
Resection of lung lesions & 5 \\
Pleural biopsy & 5 \\
Pericardial window & 2 \\
Recurrent lymphoma & 18 \\
Different diagnosis from initial lymphoma & 17 \\
Broncogenic carcinoma & 1 \\
Tuberculosis & 3 \\
Lung fibrosis & 2 \\
Interstitial pneumonitis & 3 \\
Nonspecific granuloma & 3 \\
Celomatic cyst & 1 \\
Reactive lymphnodes & 4 \\
\hline
\end{tabular}

neoplastic mass and the reactive fibrosis induced by chemotherapy and radiation therapy cause displacement and tight adhesions of mediastinal structures, and the walls of the vessels are often stiff and fragile. It is important, however, to resect and examine as much tissue as possible to rule out any residual foc of malignancy. Furthermore, these patients may have multiple lesions, and it is necessary in these cases to employ a surgical approach that makes it possible to reach them all.

From a clinical standpoint, these patients are often in poor general condition after chemotherapy and radiation therapy. If either mediastinoscopy or anterior mediastinotomy is adequate to control the lesions, it can be employed safely, ${ }^{4}$ but if a more invasive operation (such as thoracotomy or sternotomy) is required, the benefit of positive diagnosis should be carefully weighted against the increased risks of the procedure. Before the advent of
VATS, many patients could not be treated surgically because of poor general conditions and excessive risks. Our experience shows that VATS represents the solution to this problem in many cases.

Mediastinoscopy is the technique of choice for lesions located within the reach of the mediastinoscope, but this approach can be difficult and hazardous in the restaging of lymphoma because of displacement of mediastinal structures as a result of radiation therapy and chemotherapy. In addition, only small biopsy samples can be taken through this route. Anterior mediastinotomy is an excellent biopsy route, ${ }^{3,4}$ but it has limited application in a setting in which the disease may be heterogeneously distributed.

More invasive procedures such as sternal split, sternotomy, or thoracotomy, which we performed in the past, ${ }^{5}$ should be considered only when VATS is not feasible. We believe that VATS is useful in this clinical setting because severely compromised patients can also benefit from positive diagnosis and thus receive appropriate therapy.

\section{REFERENCES}

1. Filly R, Blank N, Castellino RA. Radiologic distribution of intrathoracic disease in previously untreated patients with Hodgkin's disease and non-Hodgkin lymphoma. Radiology 1976;120:277-81.

2. Mauch P, Goodman R, Hellman S. The significance of mediastinal involvement in early stage Hodgkin's disease. Cancer 1978;42:1039-45.

3. Elia S, Cecere C, Giampaglia F, Ferrante G. Mediastinoscopy versus anterior mediastinotomy in the diagnosis of mediastinal lymphoma: a randomized trial. Eur J Cardiothorac Surg 1994;57:992-5.

4. Rendina EA, Venuta F, De Giacomo T, Ciriaco P, Ricci C. Comparative merits of thoracoscopy, mediastinoscopy and anterior mediastinotomy for mediastinal biopsy. Ann Thorac Surg 1994;57:992-5.

5. Ricci C, Rendina EA, Venuta F, Pescarmona EO, Di Tolla R, Ruco LP, et al. Surgical approach to isolated mediastinal Iymphoma. J Thorac Cardiovasc Surg 1990;99:691-5.

\title{
NATIVE CARDIECTOMY IN A HETEROTOPIC HEART TRANSPLANT RECIPIENT
}

\author{
Si M. Pham, MD, Robert L. Kormos, MD, and Bartley P. Griffith, MD, Pittsburgh, Pa.
}

Heterotopic heart transplantation is traditionally indicated when the recipient has irreversible pulmonary hypertension and when the donor heart is believed to be too small to support the recipient's circulation. In long-term

From the Department of Surgery, University of Pittsburgh School of Medicine, Pittsburgh, Pa.

Received for publication Feb. 1, 1996; accepted for publication Feb. 9, 1996.

J Thorac Cardiovase Surg 1996;112:1109-11

Copyright (c) 1996 by Mosby-Year Book, Inc.

$0022-5223 / 96 \$ 5.00+0 \quad \mathbf{1 2 / 5 4 / 7 2 6 5 8}$ follow-up, mitral and tricuspid regurgitation in the native heart are commonly observed after heterotopic heart transplantation. ${ }^{1,2}$ Regurgitation of the native aortic valve is rare, however, and usually mild. We report a case of severe native aortic and mitral valve regurgitation resulting in refractory congestive heart failure in a heterotopic heart transplant recipient. The heart failure resolved after a native cardiectomy.

A patient with ischemic cardiomyopathy who had previously undergone coronary artery bypass grafting in 1970 received a heterotopic heart transplant in July 1990 at the age of 60 years. During the heart transplant operation, a piece of Dacron polyester fabric graft was used to connect 